\title{
BMJ Open Community engagement approaches for malaria prevention, control and elimination: a scoping review protocol
}

\author{
Kiran Raj Awasthi (D) , Jonine Jancey, Archie C A Clements, Justine E Leavy
}

To cite: Awasthi KR, Jancey J, Clements ACA, et al. Community engagement approaches for malaria prevention, control and elimination: a scoping review protocol. BMJ Open 2021;11:e049812. doi:10.1136/ bmjopen-2021-049812

- Prepublication history for this paper is available online. To view these files, please visit the journal online (http://dx.doi. org/10.1136/bmjopen-2021049812).

Received 02 February 2021 Accepted 24 September 2021

Check for updates

(c) Author(s) (or their employer(s)) 2021. Re-use permitted under CC BY-NC. No commercial re-use. See rights and permissions. Published by BMJ.

Curtin School of Population Health, Curtin University-Perth Bentley Campus, Perth, Western Australia, Australia

Correspondence to

Dr Kiran Raj Awasthi;

kiran.awasthi@curtin.edu.au

\section{ABSTRACT}

Community engagement (CE) is important for malaria prevention, control and ultimately elimination. As the decline of malaria has plateaued over the last 5 years, strengthening CE approaches will be necessary to enhance health promotion practice and policy to drive malaria transmission down further. Countries have adopted a wide range of public health intervention approaches for malaria prevention and control that best suit their context. This review will examine the existing evidence on the various CE approaches adopted by malaria programmes across the world and their outcomes.

Methodology and analysis The review methodology will follow the updated Joanna Briggs Institute guide for scoping review, 2017, which is based on the framework developed by Arksey and O'Malley and further developed by Levac Colquhoun and O'Brien. Proquest, Web of Knowledge and Medline will be searched for publications from January 2000 to 31 March 2021 while Google search engine will be used to find any grey literature. The eligibility criteria will be as follows: review will include primary studies written in the English language using appropriate study designs and methods, including quantitative, qualitative and mixed methods designs; and case, programme or project reports. Information on CE approaches designed specifically for malaria prevention, control, elimination and their outcomes will be explored. Subheadings and free text terms for 'community engagement' and 'malaria' will be used for the search. The article screening and data extraction will be examined by two reviewers after the initial search, and any disputes will be resolved by a third reviewer through discussion. The Preferred Reporting Items for Systematic Reviews and Meta-Analyses extension for scoping reviews guide will be used to present the review methods and the results from the search. The scoping review results will identify and map the available evidences, sources of information and research gaps in the area of CE as one approach for malaria prevention, control and/or elimination.

Ethics and dissemination This study only aims to review secondary sources and does not require human research ethics committee approval. The findings of the scoping review will be submitted to a peer-reviewed journal for wider dissemination.

\section{INTRODUCTION}

Malaria, a vector-borne disease, remains a major public health challenge contributing to an estimated 228 million cases and

\section{Strengths and limitations of this study}

To our knowledge, this is the first scoping review to be undertaken on community engagement (CE) approaches for malaria prevention, control and elimination.

- The University Health Science reference librarian will assist in developing a search strategy for the scoping review, a strength of the study.

- The review will include peer-reviewed published primary sources in English; therefore, publications in languages other than English, unpublished articles and multi-country studies will be excluded, a limitation of this study.

- As this will be a scoping review, the study will be limited to providing existing evidence on the topic with an aim to identify and conduct a narrative synthesis of the various CE approaches.

400000 deaths annually worldwide. ${ }^{1}$ Globally, between 2010 and 2014, there was a $70 \%$ decrease in malaria incidence, however, in the last 5 years, the progress towards further reduction has been relatively static. ${ }^{2}$ The earlier decrease in cases was attributed to scaling up of routine interventions such as free distribution of long-lasting insecticidal nets (LLINs) or insecticide treated nets, periodic indoor residual spraying (IRS), prompt treatment of diagnosed cases and use of artemisinin-based combination therapy for the treatment of Plasmodium falciparum malaria. ${ }^{13-5}$ While some countries focus their strategies on malaria prevention by enabling and promoting use of LLINs/IRS/larvicides and chemoprophylaxis alongside malaria control programmes that target a reduction in the disease burden to a level where it is no longer a public health concern; countries with fewer malaria cases aim for elimination to ensure sustained zero local transmission of malaria in the population within a set geographic boundary through a strengthened surveillance system. ${ }^{1}$

Community engagement (CE) is defined as 'a process of working collaboratively with 
groups of people who are affiliated by geographic proximity, special interests, or similar situations, with respect to issues affecting their wellbeing' (p9). ${ }^{6} \mathrm{CE}$ has been adopted especially by lower and middle income countries (LMICs) in a quest to reach elimination of malaria by 2030 , consistent with the WHO Global Malaria Strategy 2016-2030. ${ }^{78}$ $\mathrm{CE}$ has been used to codesign public health interventions and approaches for prevention and control of malaria in a variety of countries in a range of national programmes, such as: mass drug administration for malaria prevention in Myanmar and Laos ${ }^{910}$; increasing the use of LLINs and promoting early testing and treatment in Cambodia and Kenya $^{11}$; and improving access to diagnosis and treatment in communities in Zambia. ${ }^{12} \mathrm{~A}$ variety of activities have been implemented for malaria prevention, control and elimination based on CE. These include formation of community leadership groups comprising local decisionmakers, elderly and youth; drama campaigns and health education programmes conducted in local languages and delivered in schools and churches; house-to-house visits by community health volunteers to improve early detection and timely treatment in rural areas with high levels of migration; and participatory action malaria research led by the community. ${ }^{9-12}$

Health interventionists use CE to harness communities in health promotion practice, research and policy-related decision-making to advance knowledge and support behavioural and environmental change to improve health outcomes. ${ }^{13}$ Public health interventions can incorporate CE in different forms: providing information, consulting, joint decision-making, acting collaboratively and supporting the community interests independently. ${ }^{14} \mathrm{CE}$ can be effective in dealing with health inequalities especially among disadvantaged groups who are challenged by structural, geographical, cultural, financial and language barriers. ${ }^{15}$ Internationally, a range of CE approaches that best suit the context and the target community have been used to raise awareness of malaria prevention and enable year-round round access to free testing and treatment in rural hard-to-reach populations, while developing local level ownership. For example, in Malawi, the communitybased health animators (volunteers who conduct peer education in Malawi) have been used by the national malaria programme as peer influencers to improve awareness and promote positive behaviour change in the community. ${ }^{16}$ In Nigeria, integrated community case management has been used to detect and treat malaria cases in remote areas using trained local community health workers, minimising travel time and the cost for patients. ${ }^{17}$ Similarly, in Cameroon and Cambodia, local volunteers and village malaria workers have been used to conduct proactive and reactive case detection in communities to prevent transmission, ${ }^{11}{ }^{18}$ while the Interactive Malaria Awareness Program in South Africa has successfully used home-based care workers to form local-level partnerships and to also educate communities on malaria prevention and control. ${ }^{19}$ All these different CE approaches have contributed to improved awareness, early detection of cases and improved access and wider community acceptance of malaria prevention and treatment in the afore-mentioned countries. ${ }^{16-19}$

This paper describes the protocol for a scoping review that aims to describe CE approaches targeting the prevention, control or elimination of malaria that have been/or are being implemented by countries.

\section{REVIEW OBJECTIVES}

The objectives of the review are to map the available evidence on the types of CE approaches for (1) malaria prevention, (2) malaria control, (3) malaria elimination and (4) to describe the outcomes of the CE approaches.

\section{METHODS}

\section{Protocols and registration}

During a preliminary search, a 2016 systematic review was found that focused on one element of malaria prevention (https://doi:10.1186/s12936-016-1593-y: Malaria Journal).$^{20}$ However, no scoping review on CE has been conducted to date that incorporates different approaches to all components of malaria prevention, control and elimination across countries.

\section{Patient and public involvement}

No patient involved.

\section{Eligibility criteria}

The review will only consider intervention studies published from 2000 onwards till the end of March 2021, a period encompassing two important landmarks, the advent of the Millennium Development Goals (2000-2014) and the Sustainable Development Goals $(2015-2030){ }^{21}$

The evidence will be included if the sources are:

- primary studies.

- Written in the English language.

- Using appropriate study designs and methods, including quantitative, qualitative and mixed methods designs; and case, programme or project reports.

- Providing information on CE approaches designed specifically for malaria prevention, control and/or elimination.

The evidence will be excluded if the sources are:

- Secondary studies including systematic reviews.

- Published in languages other than English.

- Providing information on CE approaches for diseases or health issues other than malaria.

- Multicountry studies will be excluded.

- Providing anecdotal evidence without a description of the study design and methods.

\section{Information sources and search}

The search strategy will involve searching the databases for peer-reviewed published literature focusing on $\mathrm{CE}$ approaches conducted for malaria prevention, control or elimination. The search methodology will follow the 
updated Joanna Briggs Institute guide for scoping reviews in $2017,{ }^{22} 23$ which is based on the framework developed by Arksey and $\mathrm{O}^{\prime}$ Malley ${ }^{24}$ and further developed by Levac et al. ${ }^{25} \mathrm{~A}$ scoping review is a valid process of synthesising evidence on a given topic, providing an excerpt of the volume of the literature or studies without seeking to analyse it. ${ }^{26}$ Primarily, an exploratory approach, scoping reviews can shed light on the types of evidence available, the way studies have been conducted and help identify and map the evidence that is available in the area of interest. ${ }^{26} 27$

Databases including Proquest, Web of Science and Medline (OVID) will be searched using key words: "community engagement" OR "community participation" OR "community involvement " OR "public engagement" OR "community mobilization" OR "social mobilization" OR "community action" OR "community empowerment" OR "community led" OR "community conversation" AND "prevention", "control", "elimination" AND "malaria" . Similarly, advanced Google search will be used to identify grey literature including case, program or project reports using the same key words. The key words have been defined based on the objectives of the study. Initially, the search will be limited to the article title and abstract for studies published between January 2000 and 31 March 2021. The search will be further streamlined by searching for citations from the reference lists of papers selected from the initial search. For papers not available online, the first author (KRA) will contact the lead author of the publication via email requesting a copy of the paper to review.

\section{Selection of sources of evidence}

The title and the abstract obtained from the search results will be examined by two reviewers after the initial search. In the first stage of the study selection, two reviewers (JJ and JEL) independent of one another will examine the title and abstract from search results obtained by KRA. The selection of the final studies will be agreed on by three reviewers (KRA, JJ and JEL). During the final selection process, any differences regarding inclusion and exclusion of papers among the three reviewers will be discussed, and a fourth reviewer (ACAC) will be called on to reach consensus.

\section{Data charting process}

The data charting process will map the findings according to the attributes: author, date/year of publication, country/ site, aim/objectives, study population, sample size, study design, phases (prevention, control, elimination) and outcomes. The charting will be undertaken by KRA and will be reviewed by two reviewers (JJ and JEL). Any disputes or differences will be resolved by the fourth reviewer (ACAC).

\section{Presentation of the results}

The Preferred Reporting Items for Systematic Reviews and Meta-Analyses extension for scoping reviews extension for scoping reviews will be used to present the review methods and the search results. ${ }^{28}$ The 22 items checklist for reporting systematic reviews comprising two optional items (critical appraisal of sources and summary of the evidences) will be followed. The items include eligibility criteria, the search approach, methods of selecting the evidence and the data charting process. The search process and the evidence flow across various stages of the study will be presented visually using an additional diagram. Furthermore, the selected evidence based on the source, study characteristics and the major findings will be mapped and presented in tabular form. The results will be synthesised in congruence with the scoping review objectives and a narrative description will be presented. The main findings will be synthesised to highlight the limitations and provide an analysis of CE approaches paving the way for future research opportunities.

\section{Expected results}

This scoping review aims to identify the available evidence, sources of information and research gaps in the area of $\mathrm{CE}$ as one approach for malaria prevention, control and/ or elimination. The results from this review will inform future practice and research in this area.

\section{ETHICS AND DISSEMINATION}

This study only aims to review the secondary sources and does not require human research ethics committee approval. Nonetheless being a component of a mixed methods study, human ethics approval has been obtained from Nepal Health Research Council (ERB 632/2020, reference number 1287) and Curtin University's Human Research Ethics Committee number HRE2020-0701. The findings of the scoping review will be submitted to a peerreviewed journal for wider dissemination.

Twitter Jonine Jancey @joninejancey and Justine E Leavy @leavy_justine

Acknowledgements The authors would like to acknowledge the input of Vanessa Varis, Health Sciences reference librarian for her support in the designing the initial search strategy.

Contributors KRA conceptualised the topic and wrote the original draft. JEL, ACAC and JJ supervised the writing process and were involved in the review and editing of the manuscript.

Funding The authors have not declared a specific grant for this research from any funding agency in the public, commercial or not-for-profit sectors.

Competing interests None declared.

Patient and public involvement Patients and/or the public were not involved in the design, or conduct, or reporting, or dissemination plans of this research.

Patient consent for publication Not applicable.

Provenance and peer review Not commissioned; externally peer reviewed.

Open access This is an open access article distributed in accordance with the Creative Commons Attribution Non Commercial (CC BY-NC 4.0) license, which permits others to distribute, remix, adapt, build upon this work non-commercially, and license their derivative works on different terms, provided the original work is properly cited, appropriate credit is given, any changes made indicated, and the use is non-commercial. See: http://creativecommons.org/licenses/by-nc/4.0/.

ORCID iDs

Kiran Raj Awasthi http://orcid.org/0000-0001-6448-7696 
Justine E Leavy http://orcid.org/0000-0001-8747-0424

\section{REFERENCES}

1 World Health Organization. World malaria report 2019. Geneva: WHO, 2019.

2 Panda S, Swaminathan S, Hyder KA, et al. Drug resistance in malaria, tuberculosis, and HIV in South East Asia: biology, programme, and policy considerations. BMJ 2017;358:j3545.

3 World Health Organization. World malaria report 2018. Geneva: WHO, 2018.

4 World Health Organization. WHA68 2: global technical strategy and targets for malaria 2016-2030. World Health Organization, 2015.

5 Bhatt S, Weiss DJ, Cameron E, et al. The effect of malaria control on Plasmodium falciparum in Africa between 2000 and 2015. Nature 2015;526:207-11.

6 Control for Disease Control and Prevention. Principles of community engagement. CDC/ATSDR Committee on community engagement Atlanta, GA; 1997

7 Epidemiology and Disease Control Division. Nepal malaria strategic plan 2014-2025. Department of Health Services, 2016.

8 World Health Organization. Global technical strategy and targets for malaria 2016-2030. Geneva: WHO, 2015.

9 Adhikari B, Pell C, Phommasone K, et al. Elements of effective community engagement: lessons from a targeted malaria elimination study in Lao PDR (Laos). Glob Health Action 2017;10:1366136.

10 Kajeechiwa L, Thwin MM, Nosten S, et al. Community engagement for the rapid elimination of malaria: the case of Kayin state, Myanmar Wellcome Open Res 2017;2:59.

11 Lim R, Tripura R, J Peto T, et al. Drama as a community engagement strategy for malaria in rural Cambodia. Wellcome Open Res 2017;2:95.

12 Gordon A, Vander Meulen RJ, Maglior A. The 2019 Isdell:Flowers Cross Border Malaria Initiative Round Table: community engagement in the context of malaria elimination. Malar J 2019;18:432.

13 Wallerstein N, Minkler M, Carter-Edwards L. Improving health through community engagement, community organization, and community building. Health behavior: theory, research and practice 2015;5.

14 O'Mara-Eves A, Brunton G, Oliver S. The effectiveness of community engagement in public health interventions for disadvantaged groups: a meta-analysis. BMC Public Health 2015;15:1-23.
15 Cyril S, Smith BJ, Possamai-Inesedy A, et al. Exploring the role of community engagement in improving the health of disadvantaged populations: a systematic review. Glob Health Action 2015;8:29842.

16 van den Berg $\mathrm{H}$, van den Berg $\mathrm{H}$, McCann RS. The role of health animators in malaria control: a qualitative study of the health animator ( $\mathrm{HA}$ ) approach within the Majete malaria project (MMP) in Chikwawa district, Malawi. BMC Health Serv Res 2019;19:1-16.

17 Alegbeleye A, Dada J, Oresanya O, et al. Community engagement and mobilisation of local resources to support integrated community case management of childhood illnesses in niger state, Nigeria. $J$ Glob Health 2019;9:010804.

18 Bekolo CE, Williams Thomas D'Arcy, Williams TDA. Adding proactive and reactive case detection into the integrated community case management system to optimise diagnosis and treatment of malaria in a high transmission setting of Cameroon: an observational quality improvement study. BMJ Open 2019;9:e026678.

19 Cox SN, Guidera KE, Simon MJ, et al. Interactive malaria education intervention and its effect on community participant knowledge: the malaria awareness program in Vhembe district, Limpopo, South Africa. Int Q Community Health Educ 2018;38:147-58.

20 Adhikari B, James N, Newby G, et al. Community engagement and population coverage in mass anti-malarial administrations: a systematic literature review. Malar J 2016;15:523.

21 Sachs JD. From millennium development goals to sustainable development goals. The Lancet 2012;379:2206-11.

22 Peters M, Godfrey C, Mclnerney P. Methodology for JBI scoping reviews. The Joanna Briggs Institute reviewers manual 2017. The Joanna Briggs Institute, 2017: 3-24.

23 Peters MDJ, Godfrey CM, Khalil H, et al. Guidance for conducting systematic scoping reviews. Int J Evid Based Healthc 2015;13:141-6.

24 Arksey H, O'Malley L. Scoping studies: towards a methodological framework. Int J Soc Res Methodol 2005;8:19-32.

25 Levac D, Colquhoun H, O'Brien KK. Scoping studies: advancing the methodology. Implement Sci 2010;5:69.

26 Munn Z, Peters MDJ, Stern C, et al. Systematic review or scoping review? guidance for authors when choosing between a systematic or scoping review approach. BMC Med Res Methodol 2018;18:1-7.

27 Pham MT, Rajić A, Greig JD, et al. A scoping review of scoping reviews: advancing the approach and enhancing the consistency. Res Synth Methods 2014;5:371-85.

28 Tricco AC, Lillie E, Zarin W, et al. PRISMA extension for scoping reviews (PRISMA-ScR): checklist and explanation. Ann Intern Med 2018;169:467-73. 